\title{
Evacuateurs de crues en marches d'escalier
}

\author{
A. Goubet \\ Ingénieur Général des Ponts et Chaussées \\ Président du projet national $\mathrm{BaCaRa}$
}

Les barrages en béton, qu'il s'agisse de poids ou de voûtes, sont aujourd'hui généralement considérés comme particulièrement bien adaptés au passage de crues, même importantes, à travers ou au-dessus du corps du barrage. C'est même leur grande supériorité sur les barrages en remblais qui exige des évacuateurs distincts et souvent coûteux.

Jusqu'à une époque très récente tous les coursiers des évacuateurs des barrages poids étaient aussi lisses que possible et toute l'énergie du jet devait être dissipée à l'aval.

Avec l'apparition du Béton Compacté au Rouleau (BCR) s'est fait jour une nouvelle conception, matérialisée pour la première fois au barrage d'Upper Stillwater (USA1987) : puisque le béton est mis en place en couches minces, il est facile d'organiser l'extrémité aval de ces couches en marches d'escalier sur lesquelles la lame d'eau déversée perd une partie importante de son énergie, permettant de réduire d'autant les ouvrages de dissipation au pied aval.

L'intérêt de cette disposition semble tel, qu'après avoir été reprise pour de nombreux ouvrages en BCR, elle commence à être adoptée pour des barrages en béton classique.

Certains se sont même demandés pourquoi on n'y avait pas songé plus tôt, faisant remarquer par exemple que le barrage de New Croton (USA-1906) avait un évacuateur en marches d'escalier.

Il est effectivement intéressant de se poser la question — quitte à redécouvrir l'œuf de Colomb - et de faire une petite rétrospective historique. Nous reviendrons, par ailleurs, sur le cas du barrage de New Croton.

\section{Un peu d'histoire}

Dans «Usines de Retenue - Usines de Plaine" par H. VARLET (1962) on peut lire « nous nous plaçons ici dans le cas général où le barrage n'étant pas déversoir, l'évacuateur de crue est disposé latéralement» ou encore "[le déversoir latéral] est la meilleure solution quand la topographie des lieux s'y prête : en effet quand on le peut il est préférable de ne pas utiliser le barrage pour l'évacuation des crues ".
En 1962, cette idée commençait peut-être à dater, mais on la retrouve dans « Water Power engineering " par H.K. BARRows (1943) pour les seuls barrages voûtes il est vrai : "Il est clair que la section d'un barrage voûte ne convient pas pour un déversement d'eau; et si le barrage est d'une hauteur considérable la seule disposition satisfaisante est vraisemblablement la localisation du déversoir à une extrémité du barrage ou mieux encore, si cela est possible, dans une partie de la vallée à l'écart de la structure principale. »

Dans "Utilisation des Forces Hydrauliques" par Degove et Genissieu (1946) on retrouve la même idée, s'agissant cette fois des barrages poids « $\mathrm{Si}$ le barrage de retenue n'est pas trop haut, si la gorge est assez resserrée pour rendre difficile l'évacuation du trop plein par des ouvrages latéraux, on peut avoir intérêt à faire passer l'excès d'eau par-dessus le barrage." "

Et effectivement presqu'aucun des grands barrages en béton de l'époque ne comportaient de déversoirs intégrés. Les évacuateurs de crues débitaient soit dans des galeries au rocher, soit dans des canaux latéraux à l'air libre (Sautet, Chambon, Marèges, Genissiat, pour ne citer que des ouvrages français).

Si l'on étudie attentivement les documents de l'époque il semble bien que la grosse crainte associée aux déversoirs placés au centre des barrages ait été la tenue du lit à l'aval. Dans "Engineering for dams » de CRAEGER, JustiN et Hinds (1945) on peut lire « on n'est pas sûr du comportement du lit à l'aval entre 10 et $30 \mathrm{~m}$ [de hauteur du barrage], et au-delà la situation devient vite dangereuse ". Et si l'on retenait quand même la solution du déversoir central on plaçait souvent à l'aval un contre-barrage important (par exemple on conservait le batardeau) pour créer une fosse de réception.

Ce n'est en fait que depuis 1950 ou 1960 (un peu plus tôt aux USA qu'en France semble-t-il) que les évacuateurs de crue dans l'axe des barrages-poids ont eu tendance à se généraliser, à partir du moment où l'on a mieux maîtrisé les problèmes liés à la dissipation de l'énergie à l'aval. Mais à l'époque l'idée était déjà suffisamment novatrice pour que personne ne propose en outre de créer sur le parement aval des marches d'escalier dont le coffrage n'aurait pas été aisé. 


\section{Les innovations récentes}

Le barrage d'Upper Stillwater (USA-1987) est l'un des plus importants barrages en BCR au monde et le premier réalisé par le Bureau of Reclamation. Bien que terminé en 1987 il a donné lieu à de nombreuses études approfondies dès la fin des années 1970 et a comporté plusieurs innovations (dont certaines sont d'ailleurs demeurées sans suite jusqu'à présent).

Une de ces innovations consistait à réaliser les parements amont et aval en béton extrudé par levée de $60 \mathrm{~cm}$. On pouvait donc, pour le même prix, réaliser un parement de coursier à peu près lisse - mais avec des risques de cavitation tous les $60 \mathrm{~cm}$ à l'emplacement de chaque raccord - ou un parement en marches d'escalier. Des études poussées sur modèle ont montré tout l'intérêt de cette dernière solution qui a été retenue.

Pour ce barrage de $88 \mathrm{~m}$ de haut le bassin de dissipation n'a que $9 \mathrm{~m}$ de long.

Le résultat des études a été publié dès 1982 et l'idée a été immédiatement reprise pour d'autres barrages en BCR non seulement aux USA mais aussi en Afrique du Sud, au Maroc, en France notamment. Et comme ces derniers barrages étaient de dimensions plus réduites ils ont été terminés en même temps que celui d'Upper Stillwater, voire avant.

Le tableau en annexe récapitule leurs principales caractéristiques.

Ultérieurement d'autres réalisations ont vu le jour ; en France, par exemple, barrages du Riou (1990), de Choldocogagna (1991), de la Touche Poupart (1992), de Petit Saut (1994).

Quant au barrage de M'Bali, étudié par le bureau d'études CoYNe et Bellier (République Centre Africaine 1990), il a un parement aval également en marches d'escalier bien qu'il soit réalisé en béton conventionnel.

\section{Les études sur modèle physique hydraulique}

Bien entendu ces réalisations ont donné lieu à des études très complètes sur modèles physiques, au moins pour les plus importantes d'entre elles (Upper Stillwater, Monksville, Zaaihoek, les Olivettes, Petit Saut, M'Bali...).

Il convenait en effet de déterminer l'énergie résiduelle au pied du déversoir puisque l'objectif était de réduire l'importance des ouvrages dissipateurs de l'énergie de l'eau.

\section{- Upperstillwater : 3 modèles :}

- le premier du $1 / 5$, dans un canal de $76 \mathrm{~cm}$ de large pour étudier la partie supérieure, avec le parement aval de 0,6/1 initialement prévu ;

- le second au $1 / 10$, toujours pour étudier la crête mais avec le fruit de $0,32 / 1$ finalement retenu ; le modèle inclut le point de passage du fruit de $0,32 / 1$ à celui de $0,6 / 1$ adopté pour la partie inférieure du barrage ;
- le troisième au 1/15 représentant la totalité de la section, y compris le bassin de dissipation et modélisant le déversoir sur une largeur de $18 \mathrm{~m}$.

- Monksville : 3 modèles également :

- le premier au $1 / 10$ pour étudier les 7 mètres supérieurs ; - les deux autres au 1/25 représentant l'ensemble de la chute, l'un avec un évacuateur lisse, l'autre avec un évacuateur en gradins.

- De Mist Kraal : 2 modèles :

— un modèle d'ensemble au $1 / 75$;

- un modèle au $1 / 20$ pour optimiser les dimensions des marches.

- Les Olivettes: sur modèles au 1/50 on a testé des marches de $0,6-0,9$ et $1,2 \mathrm{~m}$ de haut puis on a déterminé la forme optimale pour la crête du déversoir et le raccordement aux gradins.

- Petit Saut : 3 modèles au 1/50 avec un parement lisse, des marches de $50 \mathrm{~cm}$ et des marches de $96 \mathrm{~cm}$.

- M'Bali : 2 modèles :

- un modèle au $1 / 21$ de $90 \mathrm{~cm}$ de large représentant le barrage et le bassin aval ;

- un modèle au $1 / 40$ avec fond affouillable à l'aval.

Ces modèles ont dégagé des conclusions très largement convergentes :

- le nez des marches ne doit jamais faire saillie au-delà du profil classique des déversoirs lisses; chaque marche affleure ce profil ;

- la hauteur des marches croît progressivement de manière à passer de façon continue de la crête déversante dessinée de façon classique au parement « rectiligne » constitué de marches régulièrement disposées;

- la partie supérieure lisse du déversoir ne doit pas être trop haute pour que l'eau attaque la première marche avec une vitesse limitée;

- sur chacune des premières marches l'eau forme un rouleau à axe horizontal dans lequel se dissipe une partie de l'énergie; au bout de quelques marches de l'air est entraîné, l'eau devient "blanche " et l'épaisseur du jet émulsionné demeure ensuite à peu près constante. Ceci signifie que l'énergie reste également constante ou encore que chaque marche dissipe l'énergie correspondant à sa hauteur. Evidemment ce résultat est atteint après un nombre de marches variables en fonction, notamment, du débit par mètre linéaire de crête.

Les différents auteurs indiquent l'énergie résiduelle au pied du barrage soit en pourcentage de l'énergie potentielle de l'eau, soit en pourcentage de l'énergie qui existerait au pied d'un déversoir lisse classique (ce qui les oblige à modéliser un tel déversoir en s'efforçant de respecter tant bien que mal la rugosité !). De toutes façons il est très difficile d'estimer sur modèle l'énergie d'une eau fortement émulsionnée. 
Le principe même de la présentation du résultat sous forme d'un tel pourcentage est d'ailleurs discutable puisque celui-ci décroît automatiquement en fonction de la hauteur du barrage et n'est donc pas directement transposable. Il serait plus simple de faire état de l'énergie résiduelle au pied d'un barrage de hauteur théoriquement infinie en fonction de la pente du coursier, de la hauteur des marches et du débit linéaire, quitte à préciser par ailleurs le nombre de marches nécessaires pour obtenir une hauteur d'eau émulsionnée constante (la hauteur du coursier correspondante semble être de l'ordre de 6 fois la charge sur la crête du déversoir).

De toutes façons les résultats sont spectaculaires; les taux de dissipations au pied du coursier s'échelonnent de 60 à $95 \%$ en tenant compte également, semble-t-il, de la dissipation singulière qui s'effectue immédiatement au pied du barrage dans l'émulsion. Les ouvrages de dissipation sont sensiblement réduits et pourraient, peut-être, être supprimés dans les meilleurs des cas.

(') Je ne connais pas les études qui ont pu être effectuées pour le barrage poids-voûte sud-africain de Wolvedans (parement aval $0,5 / 1)$.

\section{Le futur - les études encore nécessaires}

On pourrait croire que la cause est entendue, que tout est connu, et que l'on dispose d'un moyen peu coûteux et bien maîtrisé pour réduire sensiblement l'importance des ouvrages de dissipation à prévoir à l'aval des barrages.

On n'est pas trop éloigné de cette situation en ce qui concerne l'analyse hydraulique. Il serait évidemment totalement inutile de réétudier un modèle physique pour chaque barrage et l'on peut envisager l'établissement prochain d'abaques faisant intervenir les différents paramètres dimensionnants (encore que l'on ait peu étudié les pentes plus raides que $0,6 / 1$ que l'on pourrait envisager soit pour des barrages poids-voûte, soit pour des barrages poids en BCR présentant un parement amont incliné et un fruit aval de l'ordre de $0,5 / 1)\left({ }^{1}\right)$.

Par contre il semble que l'on n'ait pas porté attention aux fatigues subies par le béton or celles-ci sont essentielles à connaître si l'on veut dimensionner correctement les marches, toujours réalisées en béton classique jusqu'à présent, et notamment leur liaison avec le corps du barrage lorsque celui-ci est réalisé en BCR. Doit-on prévoir des

\begin{tabular}{|c|c|c|c|c|c|c|c|c|}
\hline \multirow{2}{*}{$\begin{array}{l}\text { Barrage/Pays } \\
\text { Année achèvement } \\
\text { Upper Stillwater } \\
\text { USA/1987 }\end{array}$} & \multirow{2}{*}{\begin{tabular}{|c|}
$\begin{array}{c}\text { Hauteur sur } \\
\text { fondation }(\mathrm{m})\end{array}$ \\
88 \\
\end{tabular}} & \multicolumn{2}{|c|}{$\begin{array}{l}\text { Hauteur et Longueur } \\
\text { du coursier }(\mathrm{m})\end{array}$} & \multirow{2}{*}{\begin{tabular}{|c|}
$\begin{array}{c}\text { Débit max. } \\
\text { par } \mathrm{ml}\left(\mathrm{m}^{3}\right)\end{array}$ \\
11,6
\end{tabular}} & \multirow{2}{*}{$\begin{array}{c}\begin{array}{c}\text { Charge max. } \\
\text { sur le seuil } \\
(\mathrm{m})\end{array} \\
3\end{array}$} & \multirow{2}{*}{\begin{tabular}{|c|}
$\begin{array}{c}\text { Mode calcul } \\
\text { crue }\end{array}$ \\
PMF \\
\end{tabular}} & \multirow{2}{*}{$\begin{array}{c}\begin{array}{c}\text { Hauteur } \\
\text { marche }(\mathrm{m})\end{array} \\
0,6\end{array}$} & \multirow{2}{*}{\begin{tabular}{|l} 
Fruit déversoir \\
$0,6 / 1$ et $0,32 / 1$
\end{tabular}} \\
\hline & & 59 & 183 & & & & & \\
\hline $\begin{array}{l}\text { Grindstone Canyon } \\
\text { USA/1986 }\end{array}$ & 40 & 34 & 30 & 1 & & PMF (?) & 0,3 & $0,75 / 1$ \\
\hline $\begin{array}{l}\text { de Mist Kraal } \\
\text { Afrique du Sud/1986 }\end{array}$ & 30 & 18 & 195 & & 6,2 & PMF & 1,0 & $0,6 / 1$ \\
\hline $\begin{array}{l}\text { Zaaihock } \\
\text { Afrique du Sud/1986 }\end{array}$ & 50 & 37 & 160 & 19,5 & & PMF & 1,0 & $0,62 / 1$ \\
\hline $\begin{array}{l}\text { Monksville } \\
\text { USA/1987 }\end{array}$ & 48 & 37 & 61 & & 2,6 & PMF & 0,6 & $0,78 / 1$ \\
\hline $\begin{array}{l}\text { Lower Chase Creek } \\
\text { USA/1987 }\end{array}$ & $18\left(^{1}\right)$ & 18 & 61 & 3,3 & & 1/2 PMF & 0,6 & $0,7 / 1$ \\
\hline $\begin{array}{l}\text { Les Olivettes } \\
\text { France/1987 }\end{array}$ & 36 & 31,5 & 40 & 6,6 & 2,3 & $1 / 1000$ & 0,6 & $0,75 / 1$ \\
\hline $\begin{array}{l}\text { Ain al Koreima } \\
\text { Maroc }\end{array}$ & 26 & 22 & 30 & 6,6 & & $1 / 1000$ & & $0,6 / 1$ \\
\hline $\begin{array}{l}\text { Knellpoort } \\
\text { Afrique du Sud/1986 }\end{array}$ & 50 & 43 & & & & & & $0,6 / 1$ \\
\hline $\begin{array}{l}\text { Riou } \\
\text { France/1990 }\end{array}$ & 26 & 19 & 105 & 1,05 & 0,70 & & 0,6 & $0,6 / 1$ \\
\hline $\begin{array}{l}\text { M'Bali } \\
\text { RCA/1990 }\end{array}$ & 32,5 & 25 & $2 \times 30$ & 16 & 4 & & 0,8 & $0,8 / 1$ \\
\hline $\begin{array}{l}\text { Choldocogagnia } \\
\text { France/1991 }\end{array}$ & 35 & 32,5 & 13,5 & 2,10 & 1,05 & $?$ & 0,6 & $0,85 / 1$ \\
\hline $\begin{array}{l}\text { Wolvedans } \\
\text { Afrique du Sud/1990? }\end{array}$ & 70 & 63 & & & & & 1,0 & $0,5 / 1$ \\
\hline $\begin{array}{l}\text { La Touche Poupart } \\
\text { France/1992 }\end{array}$ & 36,5 & 33 & $2 \times 17,5$ & $\begin{array}{l}6,1 \\
4,0\end{array}$ & $\begin{array}{l}2,0 \\
1,5\end{array}$ & $\begin{array}{l}\text { PMF } \\
1 / 5000\end{array}$ & 0,6 & $0,75 / 1$ \\
\hline $\begin{array}{l}\text { Petit Saut } \\
\text { France (Guyane)/1994 }\end{array}$ & 45 & 31 & 60 & 4 & 1,5 & sans objet & 0,6 & $0,8 / 1$ \\
\hline
\end{tabular}

( ${ }^{1}$ ) Hors sol. 
armatures (présentes aux Olivettes et absentes à Upper Stillwater)?

La dissipation d'énergie se fait sans doute essentiellement au sein de l'eau (pour les marches supérieures) et de l'émulsion (pour les marches inférieures); mais il s'exerce certainement sur les faces des marches des efforts de compression et de traction (ou de "succion») selon les emplacements, efforts de caractère dynamique.

Or il convient d'être beaucoup plus prudent lorsque l'on conçoit un déversoir que lorsque l'on dessine un barrage : pour les barrages du Gage et de Tolla par exemple, on a pu tenter des voûtes hardies; un remplissage prudent de la retenue a rapidement montré que l'on avait été au-delà des limites admissibles et a permis de remédier à la situation. D'une façon générale on peut pratiquement toujours savoir, dès le premier remplissage, si la conception du barrage est ou non satisfaisante.

Pour un déversoir il n'en est rien et l'épreuve «nominale » n'aura lieu que 100,1 000, 10000 ans après la mise en service de l'ouvrage (et même bien souvent jamais si l'on s'est référé à la PMF).

Quelques exemples permettent de préciser cette idée :

- nous avons parlé ci-dessus du barrage de New Croton, parfois présenté comme un barrage avec déversoir en marches d'escalier. Cet ouvrage en maçonnerie construit entre 1892 et 1906 - et qui a détenu un certain temps le record mondial de hauteur $(91 \mathrm{~m}$ sur fondation mais seulement $53 \mathrm{~m}$ au-dessus du sol) - était en fait classique pour l'époque: barrage non déversant associé à un déversoir latéral alimentant un chenal d'évacuation parallèle à la berge. Mais comme ce chenal, taillé au rocher, avait $300 \mathrm{~m}$ de long et une forte pente, sa profondeur à l'aval était proche de $40 \mathrm{~m}$; le déversoir constituait donc par lui-même un véritable barrage-poids dont la face aval était effectivement organisée en marches de $2 \mathrm{~m}$ de haut environ.

Le déversoir a donné satisfaction pendant 49 ans. Mais en 1955 survint la plus forte crue connue, de fréquence à peu près séculaire et sensiblement inférieure à la crue de projet adaptée lors de la construction. Après passage de la crue on a constaté que le déversoir comportait plusieurs fissures traversantes amont-aval et, au niveau d'une risberne située à $17 \mathrm{~m}$ sous la crête, présentait un déplacement horizontal de presque $1 \mathrm{~cm}$. Que se serait-il passé si la crue de projet s'était effectivement produite?

- le barrage de Palagnedra, mis en service en 1952, en Suisse a fonctionné de façon satisfaisante pendant une trentaine d'années jusqu'à ce qu'une très forte crue provoque des dégâts et presque la ruine de l'ouvrage: le dessin du déversoir n'avait pas été conçu pour permettre le passage des troncs d'arbres qui l'ont obstrué;

- le barrage du Pinet, sur le Tarn, en France, a vu plus de 50 ans après sa mise en service sa cote exceptionnelle de retenue dépassée pour une crue d'un débit à peine séculaire. Là aussi la conception de l'évacuateur ne permettait pas un passage aisé des corps flottants.

Ces exemples, que l'on pourrait multiplier, montrent que, contrairement à ce qui se passe pour les barrages, le retour d'expérience n'existe pas - ou n'existe qu'à très long terme - pour les déversoirs et que ce n'est pas avant longtemps, vraisemblablement, que nous saurons si les dispositions constructives adoptées de façon empirique pour les barrages en marches d'escalier qui viennent d'entrer en service sont suffisantes ou surabondantes.

Mais pour le moment, et pour ma part, je ne serais pas prêt à donner un avis favorable à la réalisation en France, d'un ouvrage analogue, en ce qui concerne le déversoir, à Upper Stillwater.

C'est pourquoi il est envisagé, dans le cadre des travaux du Projet National BaCaRa, d'étudier sur modèle physique les efforts susceptibles de s'exercer sur les marches, mais l'étude sera certainement délicate car il faudra utiliser des capteurs susceptibles de mesurer les valeurs de crête des efforts dynamiques et non seulement leurs valeurs moyennes.

En attendant les résultats d'études de cette nature, en France ou à l'étranger, on sera conduit à adopter des dispositions constructives inutilement prudentes et coûteuses, conclusion qui ne saurait satisfaire aucun ingénieur. 